\title{
ALMOST CLASSICAL SOLUTIONS OF STATIC STEFAN TYPE PROBLEMS INVOLVING CRYSTALLINE CURVATURE
}

\author{
PIOTR BOGUSEAW MUCHA and PIOTR RYBKA \\ Instytut Matematyki Stosowanej i Mechaniki, Uniwersytet Warszawski \\ ul. Banacha 2, 02-097 Warszawa, Poland \\ E-mail: p.mucha@mimuw.edu.pl,p.rybka@mimuw.edu.pl
}

\begin{abstract}
In this note we analyze equilibria of static Stefan type problems with crystalline/singular weighted mean curvature in the plane. Our main goal is to improve the meaning of variational solutions so that their properties allow us to call them almost classical solutions. The idea of our approach is based on a new definition of a composition of multivalued functions.
\end{abstract}

1. Introduction. We study the regularity of solutions to the following constant curvature problem

$$
\operatorname{div} \partial \Omega\left(\left.\nabla_{\xi} \bar{\gamma}\right|_{\xi=\vec{n}}\right)=1 \quad \text { on } \quad \partial \Omega,
$$

where $\operatorname{div} \partial \Omega\left(\left.\nabla_{\xi} \bar{\gamma}\right|_{\xi=\vec{n}}\right)$ is the weighted mean curvature $\kappa_{\gamma}$ of an unknown surface, which is the boundary $\partial \Omega$ of a simply connected region $\Omega$. The crucial role is played here by the given function $\bar{\gamma}: \mathbb{R}^{n} \rightarrow \mathbb{R}$, which determines the character of the system (1.1). To make the problem well posed we add an extra condition prescribing the volume $|\Omega|$ of the domain $\Omega$.

The equation (1.1) can be viewed as a static case of the quasi-stationary Stefan problem with surface tension,

$$
\begin{array}{lllll}
\Delta p=0 & \text { in } \quad \Omega_{t}, & p=\kappa_{\gamma} & \text { on } & \partial \Omega_{t}, \\
\left.\Omega_{t}\right|_{t=0}=\Omega_{0}, & & \frac{\partial p}{\partial \vec{n}}=-V_{\vec{n}} & \text { on } & \partial \Omega_{t} .
\end{array}
$$

In this equation $p$ is the unknown diffusing quantity, its interpretation depends upon the physics of the problem. Let us mention that system (1.2) appears in a variety of problems. It is a model of solidification of melt, in this Stefan problem $p$ is the temperature (cf. $[\mathrm{Lu}]$ ); in the Hele-Shaw problem $p$ is the fluid pressure (see [DE]); in the tumor growth

2000 Mathematics Subject Classification: 53C21, 74E15.

Key words and phrases: Stefan type problems, crystalline curvature, compositions, meaning of solutions.

The paper is in final form and no version of it will be published elsewhere. 
model (see $[\mathrm{FR}]) p$ is the internal pressure of the proliferating tissue; in the crystal growth from vapor (see [GR1]) $p$ is the supersaturation of the diffusing water vapor.

Let us go back to the description of the content of (1.2). In this system $\Omega_{t}$ is the evolving region, whose boundary $\partial \Omega_{t}$ is free. The normal vector to $\partial \Omega_{t}$ is denoted by $\vec{n}$ and the normal speed of $\partial \Omega_{t}$ is $V_{\vec{n}}$. The standard solvability condition implies that $\left|\Omega_{t}\right|=\left|\Omega_{0}\right|$, which explains the extra requirement added to (1.1). The central point is the weighted curvature $\kappa_{\gamma}$. If the anisotropy function, $\bar{\gamma}$, and a vector field $\xi$ on a manifold $S$ are sufficiently smooth, then we set (cf. [Si])

$$
\operatorname{div}_{S} \xi=\operatorname{tr}(I d-\vec{n} \otimes \vec{n}) \nabla \xi .
$$

Thus, if $\bar{\gamma}$ and the manifold $S$ are regular enough, then $\kappa_{\gamma}$ is well defined.

We notice that (1.1) simplifies if $\Omega$ is a region in the plane,

$$
\left.\operatorname{div}{ }_{\partial \Omega} \nabla_{\xi} \bar{\gamma}\right|_{\xi=\vec{n}}=\left(\left.\frac{d}{d s} \nabla_{\xi} \bar{\gamma}(\xi)\right|_{\xi=\vec{n}}\right) \cdot \vec{\tau},
$$

where $s$ is the arc-length parameter of the curve $\partial \Omega$ and $\vec{\tau}$ is the tangent vector to $\partial \Omega$ such that the system $(\vec{\tau}, \vec{n})$ is positively oriented. In the classical, isotropic setting $\bar{\gamma}(x)$ is just $|x|$. As a result, (1.3) turns into the classical Frenet formula

$$
\kappa=\left(\frac{d}{d s} \vec{n}\right) \cdot \vec{\tau} .
$$

In this case we will obtain a circle as a solution to (1.1).

We will show how to make sense out of formula (1.1) for $\bar{\gamma}$ which is just convex. Throughout this note we restrict ourselves to the two dimensional case. At this point we note that there is an extensive literature on the problem of how to interpret $\kappa_{\gamma}$ in the case of crystalline $\gamma$, see $[\mathrm{ACM}],[\mathrm{BNP}],[\mathrm{BCN}],[\mathrm{GR}]$. The issue is the non-differentiability of $\bar{\gamma}$ as well as an implicit lack of smoothness of the unknown curve.

The authors of the papers mentioned above set up an auxiliary variational problem, which stems from the fact that we have to select a section of the subdifferential $\partial \bar{\gamma}(\vec{n})$. Our approach is completely different. In a sense, we do not define $\kappa_{\gamma}$ altogether.

We have to recall the notion of solution to (1.1). We will achieve this during the generalization of the definition of $\kappa_{\gamma}$. We are interested in $\bar{\gamma}: \mathbb{R}^{2} \rightarrow \mathbb{R}$, which is barely convex and one-homogeneous. Thus, we are permitted to write

$$
\bar{\gamma}(x)=|x| \gamma\left(\frac{x}{|x|}\right) .
$$

Since $\frac{x}{|x|}$ depends only upon the angle $\phi$ between the vector $x$ and the $x_{1}$ axis we shall subsequently write $\gamma=\gamma(\phi)$.

We shall say that $\bar{\gamma}$ is crystalline if its Frank diagram, $F_{\gamma}$, i.e.

$$
F_{\gamma}=\left\{p \in \mathbb{R}^{2}: \bar{\gamma}(p) \leq 1\right\}
$$

is convex, with Lipschitz, but not $C^{1}$ boundary.

We notice that under our assumptions $\bar{\gamma}$ defines a metric in $\mathbb{R}^{2}$, where $F_{\gamma}$ is a unit ball. The unit ball in the dual space, i.e.

$$
W_{\gamma}=\left\{x \in \mathbb{R}^{2}: \forall \vec{n} \in \mathbb{R}^{2},|\vec{n}|=1, x \cdot \vec{n} \leq \bar{\gamma}(\vec{n})\right\} .
$$


is called the Wulff shape of $\gamma$. A very nice introduction to these geometric objects and their role in modelling, especially in material sciences may be found in the book by Gurtin, [Gu].

Let us suppose that a curve $\Gamma$ we are dealing with is parameterized by the angle $\phi(s)$ and $s$ is the arc-length parameter. In addition, for a fixed $\theta \in[0,2 \pi]$, we set

$$
I(\phi)=I_{\theta}^{\gamma}(\phi)=\gamma(\phi)+\int_{\theta}^{\phi} d \psi \int_{\theta}^{\psi} \gamma(t) d t .
$$

Then $I_{\theta}^{\gamma}$ is convex as long $\bar{\gamma}$ is convex, i.e. $\gamma^{\prime \prime}(\phi)+\gamma(\phi) \geq 0$ in $\mathcal{D}^{\prime}([0,2 \pi])$ holds and the weighted mean curvature takes the following form (see [MR1, eq. (3.6)])

$$
\kappa_{\gamma}=\left(\gamma^{\prime \prime}(\phi)+\gamma(\phi)\right) \frac{d \phi}{d s}(s)=\frac{d}{d s} \frac{d}{d \phi} I_{\theta}^{\gamma}(\phi) .
$$

Hence (1.1) can be viewed as follows

$$
\frac{d}{d \phi} I_{\theta}^{\gamma}(\phi)=s-\bar{s}+s^{*} .
$$

If $\gamma$ is no longer $C^{2}$, then we generalize (1.1) by

$$
\partial_{\phi} I_{\theta}^{\gamma}(\phi) \ni s-\bar{s}+s^{*} \quad \text { a.e. }
$$

where $\partial_{\phi} I_{\theta}^{\gamma}(\phi)$ is the subdifferential of $I_{\theta}^{\gamma}$ and $\bar{s}, s^{*}$ are properly chosen constants. We refer the reader to [MR1] for more details. A solution to the inclusion (1.5) yields an angle parametrization of a curve $\Gamma$ we seek, i.e. a solution to $(1.1)$, where $\Gamma=\partial \Omega$.

Let us recall from [MR1] the result on solvability of (1.1) for any general convex $\bar{\gamma}$.

THEOREM 1.1. Let us suppose that $\bar{\gamma}: \mathbb{R}^{2} \rightarrow \mathbb{R}$ is a convex, one-homogeneous, function. Then, there exists $\varphi$ a solution to (1.1), i.e. a function satisfying (1.5). Moreover the solution defines a closed curve $\Gamma$, and it is unique up to a translation; namely it is the Wulff shape of $\bar{\gamma}$.

In the statement of Theorem 1.1 the regularity of the curve we obtain is hidden. We also have to explain what exactly we mean by a solution, this will be done in Section 3 . Certainly, the convexity assumption on $\bar{\gamma}$ implies that the solution $\Gamma$ must be convex as well. In particular, it follows that the curve is a $W_{p}^{1}$-submanifold of any $p<\infty$. However, the notion of solution used here does not seem to be satisfactory. It is just an inclusion, cf. (3.1), i.e. the solution of (1.1) is a selection of a multivalued operator, see Section 3 for more details. We would like to improve this part of the result by examining the "smoothness" of the solutions.

We expect that regularity of solutions to (1.1) should be compatible with the space regularity of parabolic problems with the elliptic operator such as the LHS of (1.1). In [MR2] we studied

$$
w_{t}=\frac{\partial}{\partial s} \frac{\partial J}{\partial \phi}\left(w_{x}\right)
$$

augmented with initial and boundary conditions. We considered there a special $J$

$$
J(\phi)=\sum_{k=0}^{3} \frac{\pi}{2}\left|\phi-\frac{3 \pi}{2}+k \frac{\pi}{2}\right| .
$$


When $J(\phi)=|\phi|$, then equation (1.6) is called the total variation flow, see [BCN], because it arises when the total variation is minimized by the steepest descent.

The non-smoothness of $J$ prompts us to replace $\frac{\partial J}{\partial \varphi}$ in (1.6) by the subdifferential $\partial J$. In order to deal with the regularity problem of (1.6) we introduced a special notion of solution, called almost classical solutions. We showed that the unique weak solution to (1.6), if the data are not too bad, is an almost classical solution, see [MR2, Theorem 2.2]. This means, in particular, that the following equality holds:

$$
w_{t}=\frac{\partial}{\partial s} \partial_{\phi} J \bar{\partial} \partial w
$$

where $\partial w$ is a generalized, multivalued derivative of $w$ and $\bar{o}$ is a composition of multivalued operators, which will be defined below in Section 2 .

We will see that these two new notions are flexible and ready to be applied here to problem (1.1). Indeed, this is exactly the content of the theorem below, which is the main result of this note.

THEOREM 1.2. The unique solution $\varphi$ of (1.1) is almost classical, i.e. the equation (1.1) is fulfilled pointwise.

This statement requires further explanations. We generalized (1.1) to make sense for merely convex $\bar{\gamma}$. However, from the point of view of Theorem 1.2 the formula (1.5) is too broad, hence we improve the meaning of solutions. On the way, the notion of the subdifferential of a convex function appeared naturally. Formula (1.7) suggests that this subdifferential is evaluated at our solution $\varphi$, which has jumps. Here comes the need of compositions $\overline{0}$. The final result of the composition is a linear function with slope one and this is exactly the content of Theorem 1.2.

The above program is performed in two stages. In Section 2 we will define the composition $\bar{o}$ rigorously, and we will provide examples of how the definition works. In the last Section 3 we will recall the notion of solution to (1.1) introduced in [MR1], and we devote ourselves to the proof of Theorem 1.2.

2. The composition $\bar{\sigma}$. We begin with the obvious task of specifying the class of operators for which the composition will be well-defined. We will present a more general setting than the one which would be sufficient to state our result. We hinted above that $\varphi$ may be interpreted as a special multifunction. Namely, we shall say that a multivalued mapping $A$ defined on an interval $\mathcal{I}$ with values in $\mathbb{R}$ is a maximal multifunction of bounded variation (maximal $B V$-multifunction for short), if it can be decomposed in the following way

$$
A=M-C,
$$

where $C$ and $M$ are increasing (non-decreasing) functions treated as maximal monotone operators. In addition, for each $x \in \mathcal{I}$ at most one of the sets $M(x), C(x)$ contains more than one point.

We can recall here the following definition of a $B V$-function. Namely, an $L_{1(l o c)^{-}}$ function $A:[a, b] \rightarrow \mathbb{R}$ is called to be of bounded variation provided that its distributional 
derivative is a Radon measure and

$$
\|A\|_{T V([a, b])}=\int_{a}^{b}|d A|
$$

is finite. The quantity $\|A\|_{T V([a, b])}$ is called the total variation of $A$, see [Ru, Chapter 8], $[\mathrm{Zi}, \S 5.1]$. As usual in that case, we have to be careful with the endpoints of the domain, which is closed.

In the following considerations it is sufficient to assume that $\phi$ we deal with is a maximal $B V$-multifunction. Further restrictions will be specified later.

Thanks to the elementary properties of monotone functions at each point of the interior of the domain of $\Omega$ there exist the left- and right-hand-side limits and they determine the jumps as follows

$$
\phi\left(s_{0}\right)=\left[\lim _{s \rightarrow s_{0}^{-}} \phi(s), \lim _{s \rightarrow s_{0}^{+}} \phi(s)\right]_{o r}
$$

for each $s \in \Omega$, where

$$
[k, l]_{o r}= \begin{cases}{[k, l]} & \text { for } k \leq l, \\ {[l, k]} & \text { for } l<k,\end{cases}
$$

For the sake of convenience of the presentation, we now define the set of singularities of a maximal monotone operator.

Definition 2.1. Let us suppose that $f:[\psi, \theta] \rightarrow \mathbb{R}$ is a maximal monotone (multivalued) operator. We assume that the set $\bigcup_{x \in[\psi, \theta]} f(x)$ is bounded. We put

$$
\mathcal{S}(f)=\{\sigma \in[\psi, \theta]: \partial f(\sigma)=[r, t] \text { and } r<t\} .
$$

We will call $\mathcal{S}(f)$ the set of singularities of $f$.

Obviously, $\mathcal{S}(f)$ is at most countable, so we shall frequently write $\mathcal{S}(f)=\left\{\sigma_{k}\right\}_{k \in \mathcal{Z}}$, where $\sigma_{k}$ denotes points of $\mathcal{S}(f)$ and $\mathcal{Z}$, the set of indices, is at most countable.

We shall distinguish a subclass of the maximal $B V$-multifunction, which is particularly suitable for our analysis. A special role in this process will be played by the sets $\Xi_{k}(\phi)$. They are pre-images of the singular set of $f$. Namely, if $\sigma_{k} \in \mathcal{S}(f)$, and $\phi:[a, b] \rightarrow[\psi, \theta]$ is a maximal $\mathrm{BV}$-multifunction, then we set

$$
\Xi_{k}(\phi)=\left\{s \in[a, b]: \phi(s) \ni \sigma_{k}\right\} .
$$

Of course $\Xi_{k}(\phi)$ is a sum of its connected components, we note, however, that each component is a closed subset of $[a, b]$, because at each point $\phi$ has one sided limits.

Definition 2.2. We say that a maximal BV-multivalued function $\phi:[a, b] \rightarrow \mathbb{R}$ is of admissible total variation (ATV for short), and we shall write $\phi \in \operatorname{ATV}[a, b]$, iff $[a, b]$ may be decomposed into a finite collections of closed intervals $I_{l}=\left[a_{l}, b_{l}\right], l=1, \ldots$, $K(\phi)$, i.e. $[a, b]=\bigcup_{l=1}^{K(\phi)} I_{k}$ such that $\left(a_{m}, b_{m}\right) \cap\left(a_{l}, b_{l}\right)=\emptyset, m \neq l$ and for each $l$ the restriction $\left.\phi\right|_{\left(a_{l}, b_{l}\right)}$ or $-\left.\phi\right|_{\left(a_{l}, b_{l}\right)}$ is a maximal monotone operator and $\left(a_{l}, b_{l}\right)$ is maximal (with respect to inclusion of sets) with this property. We note that this maximality includes the degenerate cases, i.e. we admit $a_{l}=b_{l}$. Moreover, $K(\phi)$ denotes the number of elements of the considered collection. 
The points $b_{l}=a_{l+1}$ are special, there the multifunction $\phi$ switches between being decreasing and being increasing. These may be continuity points or jumps.

In particular, it follows that for each $k \in \mathcal{Z}$ and for each connected component $[d, e]$ of $\Xi_{k}(\phi)$ there exists $\epsilon>0$ and the two conditions below are fulfilled:

(i) $\phi(s)>\sigma_{k}$ for $s \in(e, e+\epsilon)$ or $\phi(s)<\sigma_{k}$ for $s \in(e, e+\epsilon)$;

(ii) $\phi(s)>\sigma_{k}$ for $s \in(d-\epsilon, d)$ or $\phi(s)<\sigma_{k}$ for $s \in(d-\epsilon, d)$.

We want to stress that Definition 2.2 excludes BV-functions with infinitely many oscillations which accumulate at one point as in the case of the function $e^{-1 / x^{2}} \sin \frac{1}{x^{2}}$.

We are now ready to recall the multi-stage process of the definition of the composition o (see [MR2] for a special case).

DeFinition 2.3. We define the composition

$$
B \bar{\circ} A:[a, b] \rightarrow \mathbb{R},
$$

where $A:[a, b] \rightarrow[\psi, \theta]$ is an ATV function, $B:[\psi, \theta] \rightarrow \mathbb{R}$ is a multivalued maximal monotone operator.

The interval $[a, b]$ is split as follows:

$$
[a, b]=\mathcal{D}_{r} \cup \mathcal{D}_{f} \cup \mathcal{D}_{s},
$$

where

$$
\begin{gathered}
\mathcal{D}_{f}=\left\{\bigcup_{k}\left(a_{k}, b_{k}\right):\left.A\right|_{\left(a_{k}, b_{k}\right)}=\theta_{k}, \text { where } \theta_{k} \text { is a constant }\right\} ; \\
\mathcal{D}_{s}=\left\{s \in[a, b]: A(s)=\left[\sigma_{s}, \tau_{s}\right] \text { and } \sigma_{s}<\tau_{s}\right\} ; \\
\mathcal{D}_{r}=[a, b] \backslash\left(\overline{\mathcal{D}_{f}} \cup \mathcal{D}_{s}\right) .
\end{gathered}
$$

Here, $\mathcal{D}_{f}$ denotes the flat areas of $A$. In the definition of $\mathcal{D}_{f}$ each interval $\left(a_{l}, b_{l}\right)$ is understood to be maximal with respect to inclusion of sets. The set of discontinuity points of $A$ equals $\mathcal{D}_{s}$. At this point we recall that a $B V$-function admits only one type of discontinuity, i.e. the right and left side limits for all points always exist, so only 'jumps' are admitted. The regular part of $A$ in this decomposition is $\mathcal{D}_{r}$, i.e. it contains what is left after removing singularities: the flat parts and jumps.

We consider three steps of the definition corresponding to the above decomposition:

1. For $s \in \mathcal{D}_{r}$ the set $A(s)$ is a singleton, hence

$$
B \bar{\circ} A(s)=B(A(s)) \text { for } s \in \mathcal{D}_{r}
$$

is a well-defined object.

2. For $s \in \mathcal{D}_{f}$ we consider the maximal interval $\left(a_{k}, b_{k}\right)$ containing $s$ and such that $\left.A\right|_{\left(a_{k}, b_{k}\right)}=\theta_{k}$. Here, we are required to consider two sub-cases:

(a) If $B\left(\theta_{k}\right)$ is a singleton, then

$$
B \bar{\circ} A(s)=B\left(\theta_{k}\right) \text { for } s \in\left(a_{k}, b_{k}\right) .
$$

(b) If $B\left(\theta_{k}\right)=\left[u_{k}, v_{k}\right]$, then we have four further cases depending upon the behavior of $A$ in a neighborhood of $\left(a_{k}, b_{k}\right)$. For sufficiently small $\epsilon>0$ we have:

(i) $A$ is increasing: $A(s)<\theta_{k}$ for $s \in\left(a_{k}-\epsilon, a_{k}\right), A(s)>\theta_{k}$ for $s \in\left(b_{k}, b_{k}+\epsilon\right)$;

(ii) $A$ is decreasing: $A(s)>\theta_{k}$ for $s \in\left(a_{k}-\epsilon, a_{k}\right), A(s)<\theta_{k}$ for $s \in\left(b_{k}, b_{k}+\epsilon\right)$; 
(iii) $A$ is convex: $A(s)>\theta_{k}$ for $s \in\left(a_{k}-\epsilon, a_{k}\right), A(s)>\theta_{k}$ for $s \in\left(b_{k}, b_{k}+\epsilon\right)$;

(iv) $A$ is concave: $A(s)<\theta_{k}$ for $s \in\left(a_{k}-\epsilon, a_{k}\right), A(s)<\theta_{k}$ for $s \in\left(b_{k}, b_{k}+\epsilon\right)$. If (i) occurs, then we put

$$
B \bar{\circ} A(s)=x_{k}\left(s-b_{k}\right)+y_{k}\left(s-a_{k}\right) \quad \text { for } s \in\left(a_{k}, b_{k}\right),
$$

where

$$
x_{k}=\frac{u_{k}}{a_{k}-b_{k}} \quad \text { and } \quad y_{k}=\frac{v_{k}}{b_{k}-a_{k}} .
$$

If (ii) holds, then we put

$$
B \bar{\circ} A(s)=x_{k}\left(s-b_{k}\right)+y_{k}\left(s-a_{k}\right) \quad \text { for } s \in\left(a_{k}, b_{k}\right),
$$

where

$$
x_{k}=\frac{v_{k}}{a_{k}-b_{k}} \quad \text { and } \quad y_{k}=\frac{u_{k}}{b_{k}-a_{k}} .
$$

For (iii) we just put

$$
B \bar{\circ} A(s)=v_{k} \quad \text { for } s \in\left(a_{k}, b_{k}\right) \text {. }
$$

Finally, for (iv) we put

$$
B \bar{\circ} A(s)=u_{k} \quad \text { for } s \in\left(a_{k}, b_{k}\right) .
$$

3. For $s \in \mathcal{D}_{s}$, first, we note that this set consists of at most countably many points and $A(s)=\left[\alpha_{s}, \beta_{s}\right]$, where $\alpha_{s}<\beta_{s}$. If $s \notin\{a, b\}$, then we put

$$
B \bar{\circ} A(s)=\left[\lim _{t \rightarrow s^{-}} B \bar{\circ} A(t), \lim _{t \rightarrow s^{+}} B \bar{\circ} A(t)\right]_{o r} .
$$

If $s$ belongs to $\{a, b\}$, say $s=a$, (the other case, $s=b$ is the same), we note that by (2.1)

$$
A(a)=\left[\alpha_{a}, \lim _{t \rightarrow a^{+}} A(t)\right]_{o r} .
$$

Then, we put

$$
B \bar{\circ} A(a)=\bigcup_{\sigma \in A(a) \backslash\left\{\lim _{t \rightarrow a^{+}} A(t)\right\}} B(\sigma) .
$$

Let us underline that it is required to remove the point $\lim _{t \rightarrow a^{+}} A(t)$ in the definition (2.9), since this value can be related to the case $2 \mathrm{~b}$ - see examples below. The endpoints of the intervals in the set $\mathcal{D}_{f}$ are treated by (2.8). They form an at most countable set.

Now we present some illustrations of the new notion of composition. We assume below that $B, A:[0,1] \rightarrow[0,1]$ and both operators are maximal monotone.

EXAMPLE 1. Let

$$
B(t)=\left\{\begin{array}{cc}
0, & t \in[0,1), \\
{[0,1],} & t=1,
\end{array} \quad A=\left\{\begin{array}{cc}
{[0,1],} & t=0, \\
1, & t \in(0,1] .
\end{array}\right.\right.
$$

Then, we have

$$
B \bar{\circ} A(t)=t .
$$

In order to evaluate the composition we used: $2(\mathrm{bi})$ and 3 formula (2.10). See Figure 1. 


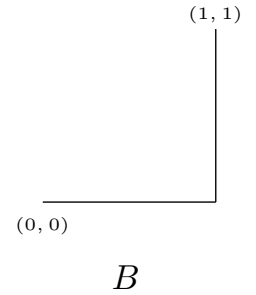

B

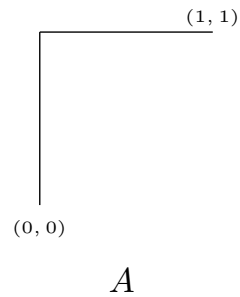

A

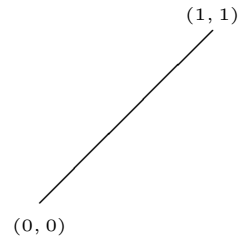

$B^{\circ} A$

Fig. 1

EXAMPLE 2. Let

$$
B(t)=\left\{\begin{array}{cc}
{[0,1 / 2],} & t=0, \\
1 / 2, & t \in(0,1), \\
{[1 / 2,1],} & t=1,
\end{array} \quad A=\left\{\begin{array}{cc}
{[0,1],} & t=0, \\
1, & t \in(0,1] .
\end{array}\right.\right.
$$

Then

$$
B \bar{\circ} A(t)=\left\{\begin{array}{cc}
{[0,1 / 2],} & t=0, \\
1 / 2(s+1), & t \in(0,1] .
\end{array}\right.
$$

Here we used: 2(bi) and 3. See Figure 2.

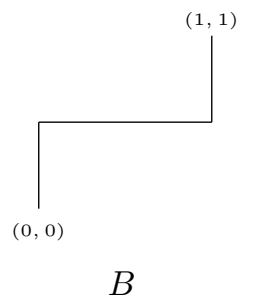

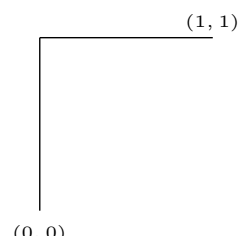

$A$

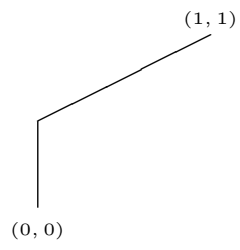

$B \bar{\circ} A$

Fig. 2

EXAMPLE 3. Let

$$
B(t)=\left\{\begin{array}{cc}
0, & t \in[0,1 / 2), \\
{[0,1],} & t=1 / 2, \\
1 & t \in(1 / 2,1],
\end{array} \quad A=\left\{\begin{array}{cc}
{[1 / 2,1],} & t=0 \\
1 / 2, & t \in(0,1 / 2] \\
t, & t \in(1 / 2,1]
\end{array}\right.\right.
$$

Then $B \bar{\circ} A(t)=1$. Here we used: 1,2 (biii) and 3 line (2.8). See Figure 3 .

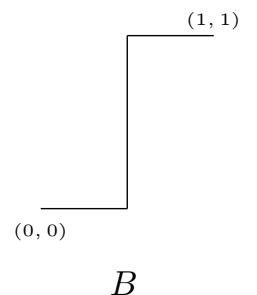

B

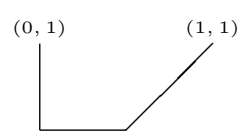

A

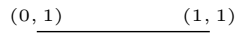

$B \bar{\circ} A$

Fig. 3 
EXAMPLE 4. Let

$$
B(t)=\left\{\begin{array}{cc}
0, & t \in[0,1 / 2), \\
{[0,1],} & t=1 / 2, \\
1, & t \in(1 / 2,1],
\end{array} \quad A=\left\{\begin{array}{cc}
t, & t \in[0,1 / 2), \\
{[0,1 / 2],} & t=1 / 2, \\
2 t-1, & t \in(1 / 2,1) \\
{[0,1],} & t=1
\end{array}\right.\right.
$$

Then

$$
B \bar{\circ} A(t)=\left\{\begin{array}{cc}
0, & t \in[0,3 / 4) \\
{[0,1],} & t=3 / 4, \\
1, & t \in(3 / 4,1) \\
{[0,1],} & t=1 .
\end{array}\right.
$$

Here we used: 1 and 3, only. See Figure 4.

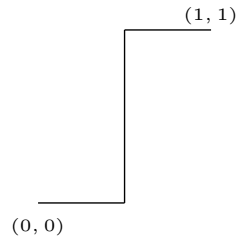

$B$

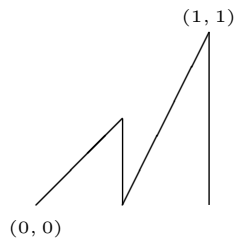

$A$

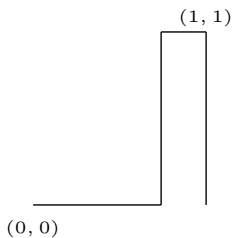

$B \bar{\circ} A$

Fig. 4

These examples suggest that the composition has good properties. Indeed, we show the following result.

Lemma 2.1. Let us suppose that $B:[\psi, \theta] \rightarrow \mathbb{R}$ is multivalued and maximal monotone, such that the set $\bigcup_{x \in[\psi, \theta]} f(x)$ is bounded. If moreover $A$ is an ATV-function, then $B \bar{\circ} A$ belongs to the ATV-class, too.

Proof. Due to the structure of functions in the ATV-class, first, we may restrict ourselves to one interval $\left[a_{l}, b_{l}\right]$ from collection for the function $A$ - see Definition 2.2. Thus $A$ is a monotone, say increasing, function on $\left[a_{l}, b_{l}\right]$. Since $A$ is increasing on $\left[a_{l}, b_{l}\right]$, it is clear that formally

$$
\|B \bar{\circ} A\|_{T V\left(a_{l}, b_{l}\right)} \leq\|B\|_{T V(\psi, \theta)} .
$$

We note that the right-hand-side above is finite, because $B$ is defined over a closed interval $[\psi, \theta]$ and by assumption $B$ takes on only finite values.

We have to make sure that $B \bar{\circ} A \in T V\left(a_{l}, b_{l}\right)$. The problem for end points $a$ and $b$ is solved by (2.9). In our case it is equivalent to verifying that $B \bar{\circ} A$ is a monotone operator. We shall show that for all $t_{1}>t_{2}$ in the domain of $A$

$$
\left(\overline{B \bar{\circ} A\left(t_{1}\right)}-\overline{B \bar{\circ} A\left(t_{2}\right)}, t_{1}-t_{2}\right) \geq 0,
$$

where $\bar{F}$ denotes an arbitrary selection of $F$.

Since $\overline{A\left(t_{1}\right)}>\overline{A\left(t_{2}\right)}$, the monotonicity of $B$ implies (2.12). The only difficulty occurs when $A\left(t_{1}\right)=A\left(t_{2}\right)=\sigma$ and $t_{1}, t_{2} \in(p, q)$ where the interval $(p, q)$ is a connected element of $\mathcal{D}_{f}$. In this case we look at part 2 of the definition. If $B(\sigma)$ is a singleton, then the 
LHS of the inequality (2.12) is zero. If not, i.e.,

$$
B(\sigma)=[r, t]
$$

then by (2.4) we calculate that

$B \bar{\circ} A\left(t_{1}\right)-B \bar{\circ} A\left(t_{2}\right)=x\left(t_{1}-q\right)+y\left(t_{1}-p\right)-x\left(t_{2}-q\right)-y\left(t_{2}-p\right)=\frac{t-r}{q-p}\left(t_{1}-t_{2}\right)>0$.

So (2.12) follows and we conclude that $B \bar{\circ} A \in B V$ on each element of the decomposition of the domain of $A$ given in Definition 2.2. The case of degenerate intervals, i.e. $a_{l}=b_{l}$ follows immediately from Definition 2.3, part 3 .

Since the behavior at the endpoints $\left\{a_{l}, b_{l}\right\}$ is controlled by the definition of the composition, (2.1) is fulfilled at these points. Hence we deduce the claim for the whole domain of the composition by combining the results for each of the intervals $\left[a_{l}, b_{l}\right]$. Thus, for a general ATV-function we have

$$
\|B \bar{\circ} A\|_{T V(a, b)} \leq K(A)\|B\|_{T V(\psi, \theta)},
$$

where $K(A)$ is the number of changes of the monotonicity of the function $A$, i.e. the number of intervals postulated in Definition 2.2. Due to the definition we have also

$$
K(B \bar{\circ} A) \leq K(A) .
$$

This finishes the proof of Lemma 2.1 .

3. Solutions to (1.1) are almost classical. We recall the notion of a solution to (1.1). According to [MR1, definition 3.1], if $\bar{\gamma}$ is one-homogeneous and convex, then by a solution to (1.1) we mean a closed curve $\Gamma$ whose angle parameterization $\varphi(\cdot)$ is a monotone (increasing) multivalued function, which can be treated locally as an $L_{1}$ function, and the differential inclusion (1.5) holds with initial data $\left.\varphi\right|_{s=\bar{s}}=\bar{\varphi}$ and $s^{\star} \in \partial_{\varphi} I_{\vartheta}^{\gamma}(\bar{\varphi})$.

We continue the discussion started at the end of Section 1. Since $\partial_{\phi} I_{\theta}^{\gamma}(\phi)$ is a maximal monotone operator, we immediately obtain a candidate for a solution, namely we find

$$
\varphi(s) \in\left(\partial_{\phi} I_{\theta}^{\gamma}\right)^{-1}\left(s-\bar{s}+s^{*}\right) .
$$

We notice that all the selections define a unique $L^{1}$ representative. Thus, since the RHS of (3.1) is ATV-function, so we may define the solution in that class by putting

$$
\varphi(s)=\left(\partial_{\phi} I_{\theta}^{\gamma}\right)^{-1}\left(s-\bar{s}+s^{*}\right) .
$$

Indeed, by [MR1, Theorem 1] the inclusion above yields the desired solution. Formula (3.1) implies that $\varphi$ is a monotone operator, and $\partial_{\phi} I_{\theta}^{\gamma}$ is a maximal monotone operator, hence the composition $\bar{\sigma}$ is applicable here. Moreover, we claim that

$$
\partial_{\phi} I_{\theta}^{\gamma} \overline{\mathrm{o}} \varphi=s-\bar{s}+s^{*} \quad \text { pointwise. }
$$

Indeed, this is the main result of this note. From now on, we shall suppress the superscript $\gamma$ and the subscript $\theta$.

THEOREM 3.1. Let us suppose that $\bar{\gamma}$ is one-homogeneous and convex, I is given by (1.4) and $\varphi$ is defined in (3.2). Then

$$
\frac{d}{d s} \partial I^{-} \varphi=1
$$


Proof. Let us set $t=s-\bar{s}+s^{*}$, in order to show $\partial I \bar{\circ} \phi(t)=t$, we consider the three cases postulated by the definition of the composition.

$1^{o}$ In the first case $t \in \mathcal{D}_{r}$. Thus, $\varphi(t)$ is a singleton, thus $(\partial I \bar{\circ} \varphi)(t)=\partial I(\varphi(t))$. In addition, $t \in \mathcal{D}_{r}$ implies that $t$ does not belong to any closed interval on which $\varphi$ is constant, i.e. there is $\epsilon>0$ such that $\left.\varphi\right|_{(t, t+\epsilon)}$ and $\left.\varphi\right|_{(t-\epsilon, t)}$ are increasing. From this, we in turn infer that $\partial I(\varphi(t))$ must be a singleton or $\partial I(\varphi(t))=\left\{I^{\prime}(\varphi(t))\right\}$ and $I^{\prime}$ is continuous at $\varphi(t)$. As a result, by the very definition of $\varphi$, we conclude that $\partial I^{-} \phi(t)=I^{\prime}(\varphi(t))=t$.

$2^{o}$ Let us suppose that $t \in \mathcal{D}_{f}$, i.e. there exists $(d, e)$ such that $\left.\varphi\right|_{(d, e)}=$ const. and $t \in[d, e]$. The very definition of $\varphi$ implies that const. $=\sigma_{k}$ for a singular value $\sigma_{k}$ of $I$ and $\partial I\left(\sigma_{k}\right)=[d, e]$. Since $\varphi$ is increasing we end up applying part 2(bi) of Definition 2.3. This, after working out the coefficients in (2.4) yields

$$
\partial I_{\bar{\sigma}} \varphi(s)=\frac{d}{d-e}(s-e)+\frac{e}{e-d}(s-d)=s \quad \text { for } s \in[d, e] .
$$

$3^{o}$ The final case corresponds to $t \in \mathcal{D}_{s}$, i.e. $\varphi(t)=[\sigma, \tau]$. But, for all elements $\rho \in[\sigma, \tau]$, by the definition of $\varphi$ we have $\partial J(\rho)=\{t\}$. Thus, by the third part of Definition 2.3, $\partial I_{\bar{o}} \varphi(t)=t$. Our claim follows.

In other words, Theorem 3.1 is equivalent to the following statement:

Let $B$ be a maximal monotone operator, $B: \mathbb{R} \rightarrow \mathbb{R}$, then $B \overline{\bar{o}} B^{-1}=I d$.

Taking the standard definition of the composition we would get: $B \circ B^{-1} \ni I d$, only. If we consider Example 1, we would get instead of $I d$ the whole square $[0,1] \times[0,1]$. This explains the hidden special character of our new composition $\bar{\sigma}$. We hope that this new approach simplifies and improves the theory.

Let us comment on another aspect of this theorem. Let us suppose that $I_{\epsilon}$ is a strictly convex regularization of $I$ and $I_{\epsilon}$ converges uniformly to $I$. We immediately notice the existence of solutions $\varphi_{\epsilon}$ to the regularized problems, i.e.

$$
\frac{d I_{\epsilon}}{d \phi}\left(\varphi_{\epsilon}(s)\right)=s-\bar{s}+s^{*}
$$

By Helly's theorem we deduce the existence of sequence $\epsilon_{k} \rightarrow 0$ such that $\frac{d I_{\epsilon_{k}}}{d \phi}(\phi) \rightarrow \Omega(\phi)$ and $\varphi_{\epsilon_{k}}(s) \rightarrow \varphi_{0}(s)$ and the convergence is for every argument. Due to the lack of continuity of $\Omega$ we cannot write

$$
\lim _{k \rightarrow \infty} \frac{d I_{\epsilon_{k}}}{d \phi}\left(\varphi_{\epsilon_{k}}(s)\right)=\Omega\left(\varphi_{0}(s)\right)
$$

However, $\Omega$ is a selection of the subdifferential $\partial I, \varphi_{0}$ is a selection of $\varphi$ and the above theorem permits us to write

$$
\lim _{k \rightarrow \infty} \frac{d I_{\epsilon_{k}}}{d \phi}\left(\varphi_{\epsilon_{k}}(s)\right)=\partial I^{-} \varphi(s) .
$$

However, it is not clear if the above identity stays true for general $\varphi$ and $I$.

Acknowledgements. This note is realized in the framework of Polish MN grant No. 1 P03A 02130 and N N201 268935. The first author has been supported by ECFP6 M.Curie ToK program SPADE2, MTKD-CT-2004-014508 and SPB-M. He thanks Laboratoire Jacques-Louis Lions, where the research was performed, for its hospitality. 


\section{References}

[ACM] F. Andreu, V. Caselles and J. M. Mazón, Parabolic Quasilinear Equations Minimizing Linear Growth Functionals, Progress in Mathematics 223, Birkhäuser Verlag, Basel, 2004 .

[BCN] G. Bellettini, V. Caselles and M. Novaga, The total variation flow in $\mathbb{R}^{N}$, J. Differential Equations 184 (2002), 475-525.

[BNP] G. Bellettini, M. Novaga and M. Paolini, On a crystalline variational problem, part II: $B V$ regularity and structure of minimizers on facets, Arch. Rational Mech. Anal. 157 (2001), 193-217.

[DE] K. Deckelnick and Ch. M. Elliott, Local and global existence results for anisotropic Hele-Shaw flows, Proc. Roy. Soc. Edinburgh Sect. A 129 (1999), 265-294.

[FR] A. Friedman and F. Reitich, On the existence of spatially patterned dormant malignancies in a model for the growth of non-necrotic vascular tumors, Math. Models Methods Appl. Sci. 11 (2001), 601-625.

[GR1] Y. Giga and P. Rybka, Quasi-static evolution of 3-D crystals grown from supersaturated vapor, Differential Integral Equations 15 (2002), 1-15.

[GR] Y. Giga and P. Rybka, Facet bending in the driven crystalline curvature flow in the plane, J. of Geometric Analysis 18 (2008), 109-147.

[Gu] M. Gurtin, Thermomechanics of Evolving Phase Boundaries in the Plane, Clarendon Press, Oxford, 1993.

[Lu] S. Luckhaus, Solutions for the two-phase Stefan problem with the Gibbs-Thomson law for the melting temperature, European J. Appl. Math. 1 (1990), 101-111.

[MR1] P. B. Mucha and P. Rybka, A new look at equilibria in Stefan type problems in the plane, SIAM J. Math. Analysis 39 (2008), 1120-1134.

[MR2] P. B. Mucha and P. Rybka, A caricature of a singular curvature flow in the plane, Nonlinearity 21 (2008), 2281-2316.

[Ru] W. Rudin, Real and Complex Analysis, 3rd ed., McGraw-Hill, New York, 1987.

[Si] L. Simon, Lectures on Geometric Measure Theory, Proc. Centre for Math. Anal. Australian Nat. Univ. 3 (1983).

[Zi] W. P. Ziemer, Weakly Differentiable Functions, Springer-Verlag, New York, 1989. 